\title{
Optimization of Circular Plate Separators with Cross Flow for Removal of Oil Droplets and Solid Particles
}

\author{
Hei $\mathrm{Ngu}^{1 *}$, Kien Kuok Wong ${ }^{2}$, Puong Ling Law ${ }^{3}$
}

\begin{abstract}
A circular gravity-phase separator using coalescing medium with cross flow was developed to remove oil and suspended solids from wastewaters. Coalescence medium in the form of inclined plates promotes rising of oil droplets through coalescence and settling of solid particles through coagulation. It exhibits $22.67 \%$ higher removal of total suspended solids (TSS) compared to separators without coalescing medium. Moreover, it removed more than $70 \%$ of oil compared to conventional American Petroleum Institute separators, which exhibit an average of $33 \%$ oil removal. The flowrate required to attain an effluent oil concentration of $10 \mathrm{mg} / \mathrm{L}\left(Q_{o 10}\right)$ at different influent oil concentrations $\left(C_{i o}\right)$ can be represented by $Q_{010} \times 10^{-5}=-0.0012 C_{i o}+0.352$. The flowrate required to attain an effluent TSS concentration of $50 \mathrm{mg} / \mathrm{L}$ $\left(Q_{s s 50}\right)$ at different influent TSS concentrations $\left(C_{i s s}\right)$ can be represented by $Q_{s s 50} \times 10^{-5}=1.0 \times 10^{6} C_{i s s}{ }^{-2.9576}$. The smallest removable solid particle size was $4.87 \mu \mathrm{m}$. Water Environ. Res., 84, 299 (2012).
\end{abstract}

KEYWORDS: circular separator, oil-water-solid separation, coalescence plates, cross flow.

doi:10.2175/106143012X13310630612646

\section{Introduction}

Effluent with a high concentration of oil droplets can be found in the oil and gas industry or shipping activities (LaRusic and Mohr, 1998). Wastewater with a high concentration of suspended solids particles is introduced to the environment from land clearing and earthworks activities resulting from erosion (Malaysia Environmental Quality Report, 2004). Regulatory requirements for oil and suspended solids content allowed in wastewater and industrial effluent discharge are $\leq 10 \mathrm{mg} / \mathrm{L}$ and $\leq 100 \mathrm{mg} / \mathrm{L}$, respectively, for most countries such as the United State, Canada, Colombia, Malaysia, and some European countries (Mohr, 2000).

The simplest systems to treat oil or solids are often inadequate; additionally, more complicated systems are either too expensive or too maintenance-intensive (Mohr, 1995). Common separators currently in use with various designs include American Petroleum Institute (API) separators, coalescing

\footnotetext{
${ }^{1}$ School of Engineering, Computing and Science, Swinburne University of Technology (Sarawak Campus), 93350, Kuching, Sarawak, Malaysia; e-mail: lngu@swinburne.edu.my.

${ }^{2}$ School of Engineering, Computing and Science, Swinburne University of Technology (Sarawak Campus), Malaysia.

${ }^{3}$ Department of Civil Engineering, University of Malaysia Sarawak, Sarawak, Malaysia.
}

plate separators, coalescing tube separators, and packing-type separators. These oil-liquid-solid separators with coalescing plates are typically rectangular (Tchobanoglous et al., 2004).

A circular separator takes advantage of the continual decrease in horizontal velocity $\left(v_{h}\right)$ as surface area increases, thereby enhancing separation. The $v_{h}$ in rectangular tanks is constant throughout. This circular oil-water-solid separator with coalescing plates is deemed suitable for small-scale wastewater flow applications for workshop, oil terminal, land clearing and earthwork surface runoff, and residential rural or low population density areas where the conveyance of wastewater to a central treatment system is costly. This separator is also suitable for wastewater treatment where availability of large areas is a constraint, such as on platforms and during shipping activities. To date, treatment technologies for small wastewater streams in terms of cost-effectiveness and local availability and serviceability of materials and equipment that require low technical skills for operation and maintenance are not readily available. A highly efficient and cost-effective oil-water-solid phase separator is deemed as an appropriate solution for water pollution control in most rural areas and in concentrated residential areas of developing countries.

There are numerous publications that address the rectangular gravity separator such as Veenstra et al. (1998), Saleh and Hamoda (1999), Foley et al. (1999), and Burns and Mohr (2000). Rommel et al. (1992) studied the droplet coalescence process and developed a theoretical design process for plate separators.

In this research, attempts were made to (a) develop a circular oil-water-solid separator with inclined coalescence plates operated at cross flows, (b) evaluate the performance of the phase separator for removal of free and physically emulsified oils and suspended solids from synthetic wastewaters, and (c) determine the required flowrate needed to attain the desired effluent oil and total suspended solids (TSS) concentration.

\section{Materials and Methods}

Generally, the mechanisms of oil-water-solid gravity separation include the principles of Stokes' Law (Perry and Green, 1997) and the boycott effect (Peacock et al., 2005). Oil and solids in water are characterized by a spectrum of droplet sizes. The droplet size that must be removed to attain a certain effluent concentration depends on the specific gravity, concentration, and average droplet size of the oil or solid (Schlegel and Stein, 2000). The sizing of an enhanced gravity separator uses the mechanism of the settling and rising velocity of the solid 